\title{
Effect of electrolytes on the electrochemical performance of nickel cobaltite-titania nanotubes composites as supercapacitive materials
}

\begin{abstract}
The effects of electrolytes on the electrochemical performance of nickel cobaltite-titania nanotubes composites as electrochemical capacitors were evaluated. Four types of electrolytes were selected to assess their effects on the prepared composites, namely aqueous electrolytes of $1.0 \mathrm{M} \mathrm{KCl}, 1.0 \mathrm{M} \mathrm{HCl}, 1.0 \mathrm{M} \mathrm{KOH}$; and an organic electrolyte, $0.27 \mathrm{M}$ tetra$n$-butylammonium tetrafluoroborate (TBATFB) ionic liquid salt in acetonitrile. The composites performed better in $1.0 \mathrm{M} \mathrm{HCl}$ and $1.0 \mathrm{M} \mathrm{KOH}$, than in $1.0 \mathrm{M} \mathrm{KCl}$ and $0.27 \mathrm{M}$ TBATFB, which suggested that aqueous electrolytes with non-neutral $\mathrm{pH}$ would improve the specific areal capacitance values of the composites. Results have shown optimal performance in $1.0 \mathrm{KOH}$, which endowed the composite with excellent rate capability up to $200 \mathrm{mV} \mathrm{s}{ }^{-1}$. Cyclic voltammogram of the composite analysed in 1.0 M KOH produced a leaf-shaped like profile, with higher current densities towards more positive potentials. Charge-discharge analyses in $1.0 \mathrm{M} \mathrm{KOH}$ has shown that the composite possessed specific areal capacitance of up to $214.76 \mu \mathrm{F} \mathrm{cm}$ when it was evaluated at the current density of $350 \mu \mathrm{A} \mathrm{cm}$. The composite also retained up to $97.79 \%$ of its specific areal capacitance when current density was increased to $400 \mu \mathrm{A} \mathrm{cm}$. This material has demonstrated potential application for electrochemical capacitors through its facile fabrication technique.
\end{abstract}

Keyword: Electrolytes; Electrochemical performance; Nickel cobaltite-titania nanotubes composites; Supercapacitive material; Electrochemical capacitor 